\title{
Development of a Non-Contact Nurse Call System by Image Processing of Eye Movement*
}

\author{
Takenao Sugi ${ }^{\dagger}$, Masaya MinodA ${ }^{\dagger}$, Yoshitaka MatsudA ${ }^{\dagger}$, Satoru Goto $^{\dagger}$, \\ Naruto Egashira ${ }^{\ddagger}$, Ayame Oishi ${ }^{\S}$ and Takao YAMASAKI ${ }^{\S}$
}

\begin{abstract}
This paper proposes a nurse call system driven by image processing of user's eye movements. The system consisting of a computer, a camera, two light emitting diode (LED) lamps and a speaker is capable of non-contact/non-constraint operation. A user moves his/her eyes according to the predetermined specific pattern, then, the system extracts those pattern of eye movements from the image. After detecting those pattern, the system makes a buzzing sound as same as the role of call button at bedside in hospital. During the system execution, a user can always grasp a current situation of the system operation as flashing pattern of two LED lamps. Construction and evaluation of the algorithm for detecting eye movement pattern from the image were done by using the data recorded from normal healthy subjects. Developed prototype system was used in a subject with movement disorders caused from nerve degeneration. Operation of the system by the subject was accomplished to be in a satisfactory level. Further improvement of the system was expected in the contribution to the communication aid for patients with various movement disorders.
\end{abstract}

\section{Introduction}

Movement disorders cause various symptoms to the difficulties in motions. Especially, progressive neurodegenerative diseases such as amyotrophic lateral sclerosis, multiple system atrophy, etc., their degree of disabilities depends on the progression of disease[1,2]. Development of communication aid devices improves the quality of daily life for such people. In the past, considerable numbers of devices for the purpose of communication aid and/or motor assistance have been developed for people with various movement disorders[3-13].

\footnotetext{
* Manuscript Received Date: June 1, 2018

This paper has already been presented in the 49th ISCIE International Symposium on Stochastic Systems Theory and Its Applications (SSS'17) which was held in December, 2017.

† Graduate School of Advanced Technology Fusion, Saga University; 1 Honjo-machi, Saga-city, Saga 840-8502, JAPAN

‡ National Institute of Technology, Kurume College; 1-11 Komorino, Kurume, Fukuoka 830-8555, JAPAN

$\S$ Department of Neurology, Minkodo Minohara Hospital; 3553 Kanaide, Sasaguri, Kasuya, Fukuoka 811-2402, JAPAN

ฯ Department of Clinical Neurophysiology, Neurological Institute, Graduate School of Medical Sciences, Kyushu University; 3-1-1 Maidashi, Higashi-ku, Fukuoka 8128582, JAPAN

Key Words: image processing, eye movement, nurse call system.
}

Types of communication aid system can be classified by what biological information is used. Devices driven by residual muscles are effective for people with mild/moderate movement disorders[3-5]. Eye movement is one of useful motion as communication way by using residual muscles[6-11]. Electrooculography is also a measurement techniques of eye movement $[6,7]$. As one of techniques for extracting human motion features, image processing has been used in the communication aid system[8-11]. Character input devices by using the change of eye-gaze direction are typical communication aid system[10,11]. However, the degree of freedom for handling the communication aid devices is dependent upon their progress of disorders. Such devices cannot work sufficiently for the people whose movement symptom is in serious condition. In most serious cases as totally locked in state (TLS), brain computer interface and/or brain machine interface is only considered to be a possible device for communication aid[12,13].

Concerning the activity of residual muscles, orbicular muscle of the eye is often movable even as their symptoms progress. Thus, even though complex and various communication is difficult, the communication aid system using eye movement is considered to be usable in a period until becoming TLS.

We have been developing a simple device for communication aid using image processing of eye movement. Final goal of this system is to use as a nurse call system as for patients with serious movement disorders at bedside in hospital. In our prior study, accuracy of the algorithm for detecting eye-gaze direction 
was verified[14]. This paper has improved the system applicable for actual usage in the hospital. Structure of the device and the detection algorithm were modified by using the data of normal healthy subjects. Then after, one of subjects with serious movement disorders cooperated with this study, and the subject tried to use the system. It was evaluated that the sufficient result was obtained as a prototype system.

\section{Method}

\section{$2.1 \quad$ Subjects}

Three normal healthy adults (male, 21-23 yo) and a subject with movement disorders (female, 64 yo) were participated. Ethics approval was obtained in the committee in Minohara Hospital, Fukuoka, Japan. After detailed explanation about the significance of this study, written informed consent was received from three normal healthy adults. For a subject with movement disorders, cooperation to this study was confirmed both from the person herself and her family.

The data of normal healthy adults was used to construct the algorithm and to evaluate the accuracy of the system. Then, a subject tried to operate the developed prototype system in the hospital.

Movement disorder of a subject was caused from a multiple system atrophy. On the day of the prototype system operation, right eye was opened and was movable, and left eye was not able to open clearly. Some nystagmus were also observed during the system operation. There were no usable residual muscles except eye movement. She could move her eyes to the left but was hard to gaze the right.

\subsection{System Structure}

Fig. 1 shows the prototype of a nurse call system developed in this study. A nurse call system consisted of a computer, a camera, two light emitting diode (LED) lamps and a speaker (Fig.1 (a)). The system took images of user's face by a camera, then extracted the characteristics of eye movements from the images. Current state of the operation in the system was indicated to a user via two LED lamps. When a predetermined specific pattern of eye movements was detected, a speaker made the buzzing sound for informing to surrounding people such nurse, caregiver and others.

The device, consisting of a camera and two LED lamps (Fig.1 (b)) was placed at the front top of a user lying down on the bed. The device was attached to the tip of arm with four-degree-of-freedom, and its position and direction could be easily adjusted. Frontal lamp and a camera were just placed at the front of a user's face. Left lamp was located at the left side from the frontal lamp. Distance between two lamps were adjustable within the range of $40 \mathrm{~cm}$. Each LED lamp flashed with different pattern according to the state of operation and also had a role for the point of gaze.

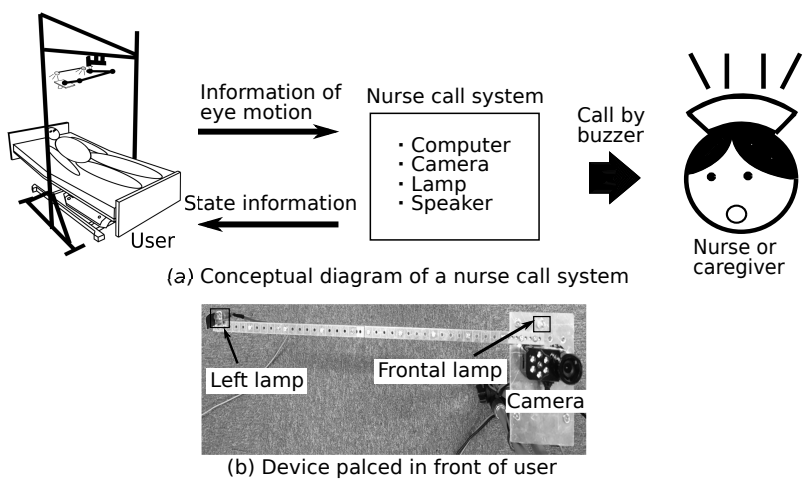

Fig. 1 Prototype nurse call system operating by image processing of eye movement

\subsection{Algorithm for Eye Movement De- tection}

Whole flowchart of eye movement detection is represented in Fig.2. In "Preprocessing" part, templates of the face and the eye of a user were created beforehand. Region of both templates were manually selected. The condition for classifying the direction of eye-gaze was also adjusted in the preprocessing part. Then after, the detection algorithm was executed. In "Image acquisition" part, images of a user were stored into the computer with the resolution of $1280 \times 780$ pixels and 5 frames per second (fps). Original color image was transformed to the grayscale one. Next in "Template matching" part, positions of the face and the eye were detected in each image by using the square difference matching method[14].

After detecting the area of user's eye by the above procedure, characteristics of eye movement were extracted. To determine the direction of eye-gaze, "Detection of pupil position" was done. Besides, to avoid the mis-detection of pupil position, "Rejection of eye blinking" was also taken into account. Based on the change over time for the detected direction of eyegaze, appearance of the specific eye movement pattern was decided in the part of "judgment of specific pattern". If the predetermined specific pattern of eye movement was detected, then a speaker made the buzzing sound.

In this study, threshold values in the algorithm were determined by using the data of normal healthy adults. How to set up the specific eye movement pattern was taken into account a patient's symptoms on the movement.

Details about the parts of "Detection of pupil position", "Rejection of eye blinking" and "Judgment of specific pattern" were described in the following sections.

\subsection{Detection of Pupil Position}

Pupil located at the center of eyeball reflects the changes in the direction of eye-gaze. Color of the pupil is dark as compared with other parts in the eye, and it can be regarded as that the pupil is lowest luminance 


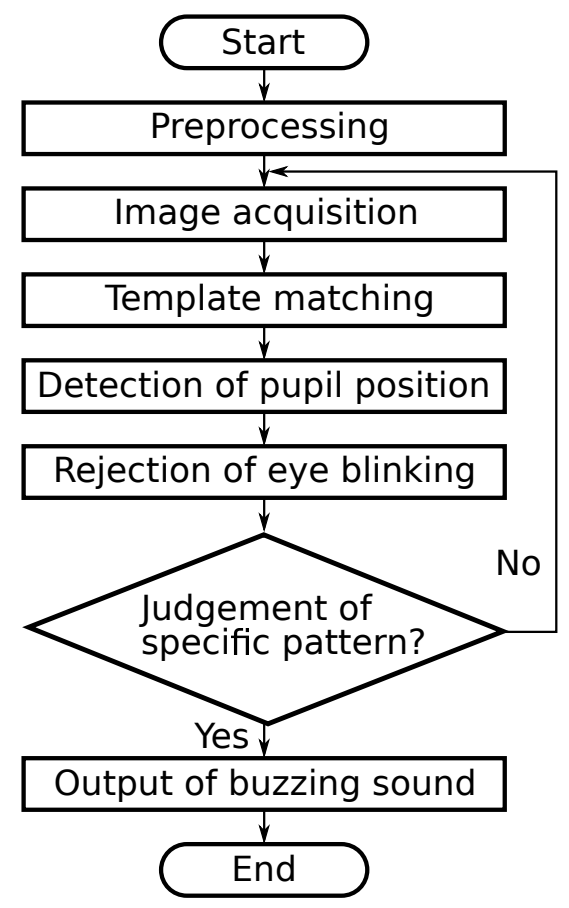

Fig. 2 Flowchart for judgment of specific eye movement pattern

in the image of eye. In this study, a method proposed by Watanabe et al.,[15] was adopted for detecting the pupil position.

At first, extracted image of eye area was segmented by the rectangular blocks of $\left(b_{x} \times b_{y}\right)$ as shown in Fig.3. Then, summation of luminance $L_{s}\left(n, x_{k}, y_{k}\right)$ for each segmented $k$ th block was calculated as follows.

$$
L_{s}\left(n, x_{k}, y_{k}\right)=\sum_{i=0}^{b_{x}} \sum_{j=0}^{b_{y}} L(n, x+i, y+j)
$$

Where, $L(n, x, y)$ means the value of luminance for pixel $(x, y)$ at $n$th frame. $x_{k}\left(=x+b_{x} / 2\right)$ and $y_{k}\left(=y+b_{y} / 2\right)$ were the center coordinate of $k$ th block. Next, the block which was minimum value of $L_{s}\left(n, x_{k}, y_{k}\right)$ out of all blocks in $n$th frame, was regarded as the region of the pupil. Its coordinate $\left(x_{k}, y_{k}\right)$ was determined as the center position of the pupil $\left(x_{c}, y_{c}\right)$.

Finally, the direction of eye-gaze was classified by the value of pupil position $\left(x_{c}, y_{c}\right)$ into three: right, front and left. If the horizontal position of pupil $x_{c}$ was below the threshold $\theta_{R}$, the direction of eye gaze was judged as right. As same way, the direction was determined as left when $x_{c}$ was larger than $\theta_{L}$. Otherwise, the direction was regarded as front.

\subsection{Rejection of Eye Blinking}

Eye blinking is a physiological phenomenon and its contamination during the system operation is unavoidable. Accurate pupil position cannot be estimated from the image data when eye blinking occurs.

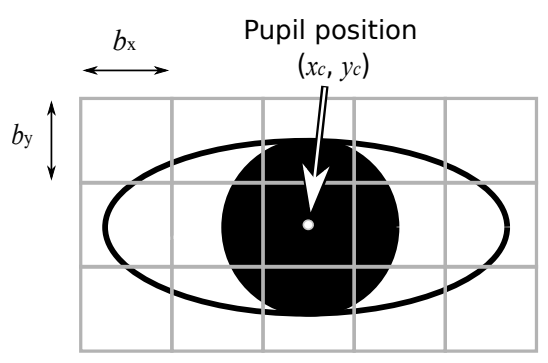

Fig. 3 Segmentation of eye image for detecting pupil position

Accordingly, the rejection of eye blinking from the image data is crucial. Eye blinking is a rapid closingand-opening motion of eyes, the characteristics of image for eye-open and eye-closed were extremely different. In addition, eye blinking was seen in the serial three to four frames ( 5 fps was the recording condition).

Based on the above features, the background subtraction method[16] was used for detecting the data which contaminated with eye blinking. The sum of differences between $n$th and $n-1$ th images was defined as $D(n)$. $D(n)$ becomes negative value if the state changes from eye-open to eye-closed, and takes positive value if the state changes from eye-closed to eye-open. Then, the following three equations were established by using $D(n)$ as

$$
\begin{aligned}
(D(n+1)-D(n))(D(n)-D(n-1)) & <0 \\
D(n) & <-\theta_{B} \\
\max \{D(n+1), D(n+2)\} & >\theta_{B}
\end{aligned}
$$

Equation (2) corresponded to the occurrence of eye blinking, eq.(3) was the state changes from eyeopen to eye-closed, and eq.(4) expressed the re-opening eyes.

If eye blinking was detected, those images were not used for detecting pupil position. In such case, the pupil position estimated from the image data which was immediately before the appearance of eye blinking was substituted instead.

In addition to the rejection of eye blinking, amendment by smoothing was also done. Actually, if the data $x_{c}$ in $n-1$ th and $n+1$ th frame were the same, and its value of $n$th frame was different, then the value of $n$th frame was amended to the same value in $n-1$ th and $n+1$ th frame.

\subsection{Judgment of Specific Pattern}

Proposed system is operated by detecting the specific eye movement pattern. Construction of such specific pattern is composed of a combination of the direction of eye-gaze: right, front and left. The pattern should not be so simple in order to avoid the missoperation, but has to be a motion that can be done by a user within low fatigue.

Based on the way of thinking, the specific pattern 


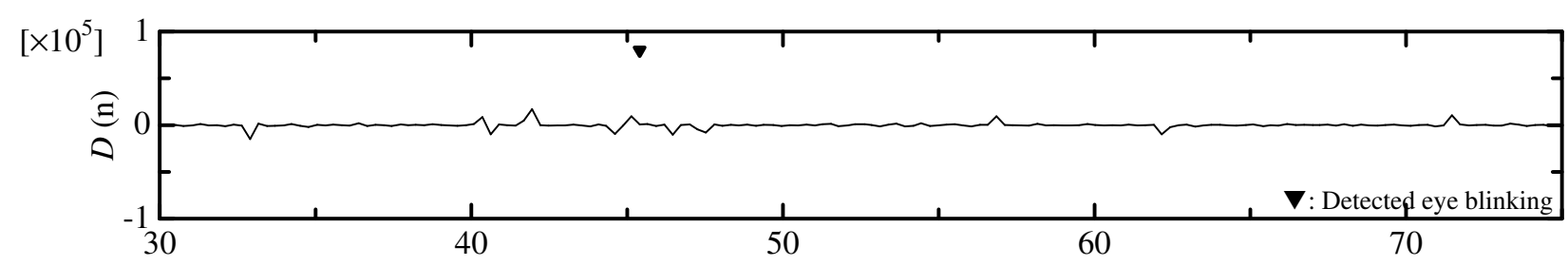

(a) Difference and detected eye blinking

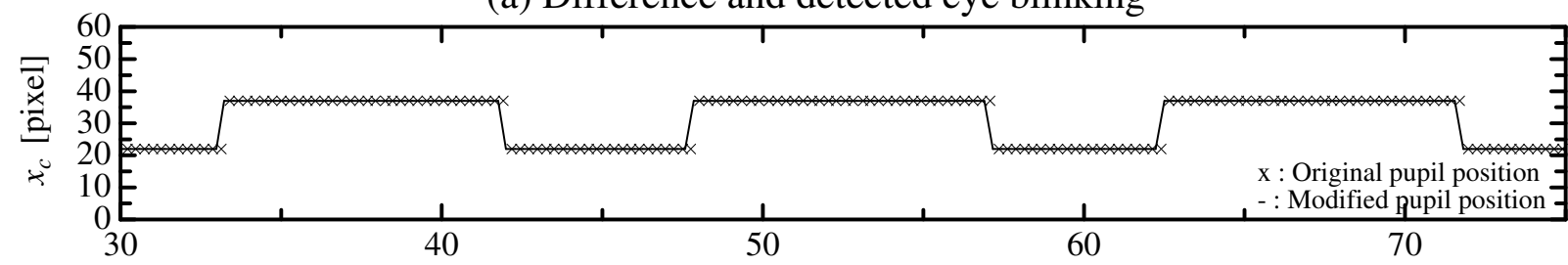

(b) Pupil position

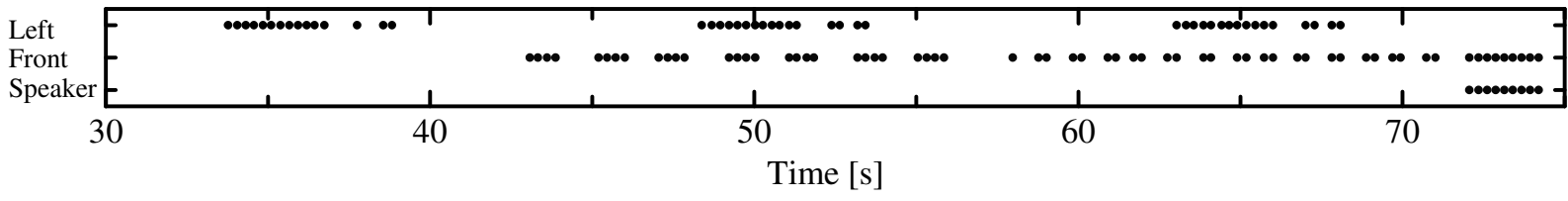

(c) State of system

Fig. 4 Result of system operation by a normal healthy subject

was defined as: if a user repeated to gaze the left target (left LED lamp) and the front target (front LED lamp) for three times alternatively, then the system made buzzing sound by a speaker. Detail procedure was in the following. First, when a user gazes at the left target, left LED lamp turns on during gazing. If a total time of gazing the left target is longer than 3 seconds (not necessary to gaze continuously), left LED lamp turns off. Next, after left LED lamp turned off, a user gazes the front target, then front LED lamp starts to flash with a cycle per second (cps). As same way of the above pattern, if a user gazes a left target longer than 3 seconds and then return to gaze the front target again, left LED lamp turns on and off, and front LED lamp starts flashing with different 0.5 cps. Finally, when a user repeats its pattern three times in total, a speaker makes a buzzing sound for 5 seconds.

The algorithm for initializing the state of operation was also defined. If a user gazed the front target continuously for 10 seconds, the system reset the operation to the initial state.

\section{Results}

\subsection{Evaluation of System Operation by Normal Subjects}

Parameters and threshold values in the algorithm were determined by the data of three normal healthy adults. Parameters $b_{x}$ and $b_{y}$ for dividing the image into segmented blocks were set at 15 . Threshold values $\theta_{R}$ and $\theta_{L}$ for classifying the direction of eye-gaze were determined at 17 and 34 , respectively. $\theta_{b}$ for detecting eye blinking was adjusted to 5000 .

Each subject executed the continuous 10 minutes operation for the system. As shown in Table 1, total
Table 1 Accuracy for detection of eye blinking

\begin{tabular}{lccc}
\hline & Correct. & F. N. & F. P. \\
\hline Sub. N1 & 35 & 2 & 0 \\
Sub. N2 & 27 & 1 & 3 \\
Sub. N3 & 24 & 0 & 2 \\
Total (N1-N3) & $86(97 \%)$ & $3(3 \%)$ & 5 \\
\hline Sub. M1 & $17(89 \%)$ & $2(11 \%)$ & 9 \\
\hline
\end{tabular}

$89(86+3)$ portions of eye blinking were found by visual inspection in the images. Eighty-six portions of eye blinking out of 89 were correctly detected by the system and $97 \%$ of the detection rate was obtained. False negatives (F. N.) and false positives (F. P.) were 3 and 5 , respectively.

During the system operation, subjects executed eye movement by their free intention without any instruction. Fig. 4 shows an example of the system operation by a subject (Subject N1, 22M). Section of 30 to 75 seconds of the data was indicated. The parameter $D(n)$ for detecting eye blinking and its result (black reversal triangular) were expressed in Fig.4 (a). Original pupil position $(\times)$ and modified pupil position (solid line) by the rejection of eye blinking were represented in Fig.4 (b). States of the left LED lamp, the front LED lamp and the speaker were noted in Fig.4 (c). First, the subject moved his eyes to the left $(33 \mathrm{~s})$, the left LED lamp turned on. Then, the direction of eye gaze was returned to the front (42 $\mathrm{s}$ ), the front LED lamp began flashing. Those operation was repeated again at 48-57 $\mathrm{s}$ and $62-72 \mathrm{~s}$. After 3 times operation, the speaker made a buzzing sound at the time of $72 \mathrm{~s}$. In case of normal healthy subjects, they had operated the system successfully 


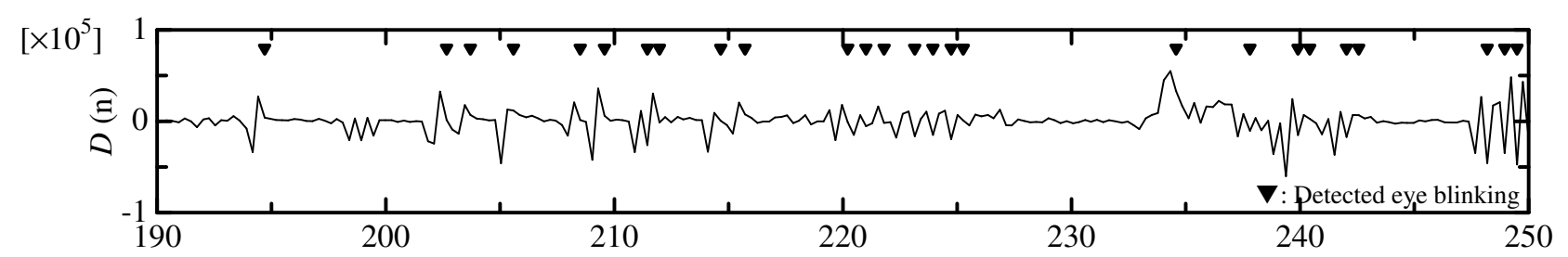

(a) Difference and detected eye blinking

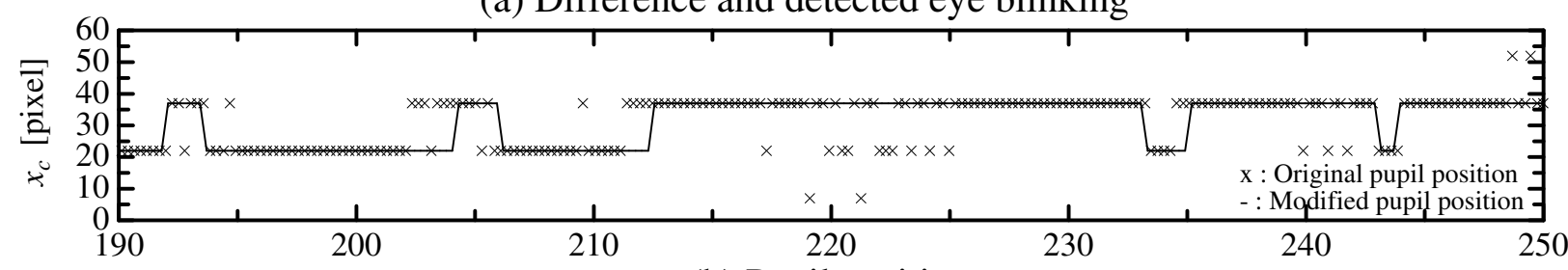

(b) Pupil position

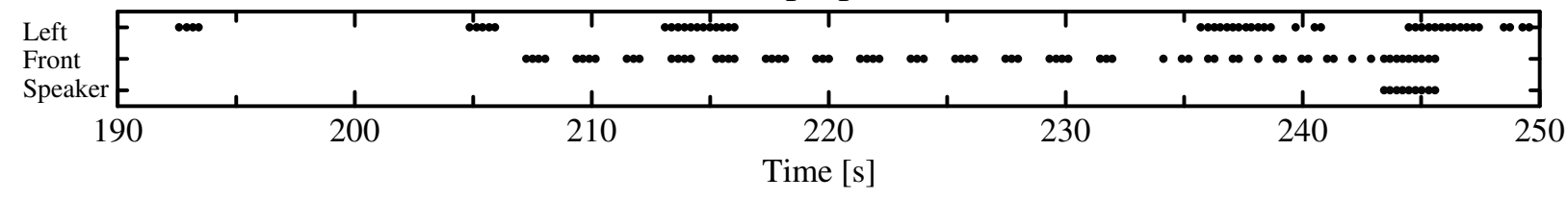

(c) State of system

Fig. 5 Result of system operation by a subject with movement disorder

without any miss-operation and confusion during continuous 10 minutes trial. Those were shown in Table 2.

\subsection{System Operation by a Subject with Movement Disorders}

A subject (Subject M1) who was hospitalizing in Minohara Hospital operated the developed prototype system. After a few trials with oral instruction, a subject executed the system operation for a continuous 10 minutes. Fig. 5 shows a part of the operation from 190 to 250 seconds. A subject gazed at the left target (192-194 s) but it was no longer than 3 seconds, consequently the front LED lamp was not flashed. A subject gazed the left target again (205-207 s). The total gazing time gained for 3 seconds or more. Then, the front LED lamp started flashing at 1 cps. A subject gazed at the left target (212-233 s) and returned its eyes to the front $(233 \mathrm{~s})$. According to those operations, the front LED lamp changed the flashing cycle to $0.5 \mathrm{cps}$. Finally for the third time, a subject gazed the left target (235-243 s), then moved its eyes to the front $(243 \mathrm{~s})$. The speaker made a buzzing sound with a successful system operation.

During the system operation from 190 to $250 \mathrm{sec}-$ onds, frequent eye blinking was observed. Twenty-six portions of eye blinking were automatically detected by the system, and 17 portions out of 26 were correctly detected. Nine false positives and 2 false negatives were obtained. An accuracy of the automatic detection was $89 \%$ (Table 1). In addition, continuous nystagmus was further seen during the system operation.

A subject has tried to operate the system for three times in 10 minutes. The result shown in Fig.5 was
Table 2 Accuracy of system operation

\begin{tabular}{lccc}
\hline & Correct. & F. N. & F. P. \\
\hline Sub. N1 & 6 & 0 & 0 \\
Sub. N2 & 7 & 0 & 0 \\
Sub. N3 & 6 & 0 & 0 \\
Total (N1-N3) & $19(100 \%)$ & $0(0 \%)$ & 0 \\
\hline Sub. M1 & $1(50 \%)$ & $1(50 \%)$ & 0 \\
\hline
\end{tabular}

the first trial. In the second trial, continuous gazing to the left target was seemed to be difficult. The operation was a failure in the middle, because the initialization of the state by gazing at the front target for 10 seconds occurred. The third trial was canceled on the way due to a subject's fatigue. Total accuracy of the system operation was $50 \%$ (1 out of 2 ) as shown in Table 2.

\section{Discussion}

\subsection{Significance of Proposed System}

A system in the past study[11] has functions for the input of Japanese letters, English letters and numerals by image processing of eye-gaze and eye blinking. It can also be used as a computer keyboard like Space, Enter and Backspace. Other studies [8-10] on character input system by eye movements also have similar functions. In contrast, the proposed system has only a function as a nurse call. The purpose of the proposed system is different for the above studies. Basic idea and techniques for extracting the features of eye movements by image processing are the same. However, target users and the situation to be used are not the same.

Nurse call system is deemed to be a simplest de- 
vice to the communication aid for disabled people. Advantages of the system proposed here are non-contact, non-constraint and capable of voluntary communication without any other person's support. More complex communication is impossible by the proposed system. However, for people with serious movement disorders such a subject who cooperated with this study, realization of spontaneous and/or voluntary communication is crucial in their daily life.

The target user for the proposed system was defined as, the people who can move their eyes but not smooth motion required, and other residual muscle is hard or impossible to move. In other words, the proposed system targets on the role of a nurse call system for people with severe movement disorders due to neurodegenerative diseases. There are various symptoms for their movement disabilities. The target users cannot move any residual muscles voluntary and spontaneously except their eyes. As a matter of fact, most of neurodegenerative diseases except ALS affect not only for movement disabilities but also other brain dysfunctions on perception, cognition and decision. In addition, those symptoms including movement disabilities vary day by day. The system development should consider the above situation. In concrete terms, robustness, adaptability and simpleand-easy operation are essential for the system improvement. The current system has not solved those items at the level of practical use.

Other communication devices by using eye movement [6-11] had achieved the intricate communication as compared with the proposed system. Those communication devices require the capability of users' motion for more than a certain degree. Adaptability for each user's motion disabilities was also not so taken into account.

In the proposed system, the placement of two LED lamps and the specific eye movement pattern were determined with the consideration of a subject's current situation for the movement disability. Adjustment of the system to the changes of the state of users' movement disabilities is capable. To increase the number of LED lamps, to replace them and to change or to modify the specific eye movement pattern are realizable.

\subsection{Enhancement of System Adaptabil- ity}

In the results of eye blinking detection, false negatives and false positives were observed both for normal healthy adults and a subject with movement disorders. In case of a subject with movement disorder, the state of the open eye was not so clear during the system operation. Therefore, some of the changes from open eye to closed eye (from closed eye to open eye) was hard to judge. Those were the reason for the false negatives. Involuntary movements of the eyelid were sometimes seen, and caused the false positives. In case of normal subjects, the cause of false nega- tives were seemed to be the low frame rate of image processing $(5 \mathrm{fps})$. The images those were extracted from the parts of false negatives were the state of half-opened eyes. False positives were caused from the movements of eyelid other than eye blinking. Revision of the algorithm is necessary for improving the accuracy of the eye blinking detection.

The result was not a perfect but was regarded as satisfactory achievement was obtained as the prototype system. The trial in the hospital was under restricted condition. The subject executed the system operation within a short period. It was considered to be a cause of fatigue. In addition, the degree of nystagmus seemed to be larger than usual. However, the above situation can be considered to occur in the system use. Classification of such nystagmus pattern and other involuntary eye movements are required for the system improvement.

Patient's posture is frequently changed in order to prevent the bedsore. The system requires the initial setting for each posture change. The initial setting needs to be done manually in the current system, but is time consuming and laborious for the actual usage. Automatic recognition of the face and the eye is also a need.

The state of eye movements changes according to the progress of the symptom and/or the physical condition of patients for the day. Auto-tuning of threshold values in the algorithm is also effective for the long-term use. Some data based learning algorithm are available.

Nurse call system should be always used during 24 hours a day. In a day, brightness around user frequently changes, and is almost dark in the nighttime. Correspondence to those things by the algorithm, the use of infrared camera etc., is also the crucial thing.

\section{Conclusions}

A nurse call system which was operated by image processing of user's eye movement was proposed. The system had a simple function for informing to the other person. The system was tested on a subject with serious movement disorders in a hospital. The subject could operate the system under the limited condition, and the result was considered to be at a satisfactory level of the first prototype system. Further development of the system will bring users with various movement disorders the improvement of their daily life.

\section{Acknowledgements}

The authors express our deepest appreciation to the staff in Minkodo Minohara Hospital for their cooperation and fruitful discussion in the development of the prototype nurse call system. 


\section{References}

[1] L. P. Rowland: Diagnosis of amyotrophic lateral sclerosis; Journal of the Neurological Sciences, Vol. 160, pp. 6-24 (1998)

[2] S. Gilman, G. K. Wenning, P. A. Low, D. J. Brooks, C. J. Mathias, J. Q. Trojanowski, N. W. Wood, C. Colosimo, A. Dürr, C. J. Fowler, H. Kaufmann, T. Klockgether, A. Lees, W. Poewe, N. Quinn, T. Revesz, D. Robertson, P. Sandroni, K. Seppi and M. Vidailhet: Second consensus statement on the diagnosis of multiple system atrophy; Neurology, Vol. 71, pp. 670-676 (2008)

[3] P. H. Veltink, B. F. J. M. Koopman, V. D. Helm and A. V. Nene: Biomechanics - Assisting the impaired motor system; Archives of Physiology and Biochemistry, Vol. 109, No. 1, pp. 1-9 (2001)

[4] J. Lobo-Prat, P. N. Kooren, A. H. A. Stienen, J. L Herder, B. F. J. M. Koopman and P. H. Veltink: Non-invasive control interfaces for intention detection in active movement-assistive devices; Journal of Neuro Engineering and Rehabilitation, Vol. 11, pp. 1-22 (2014)

[5] R. Booth and P. Goldsmith: A wrist-worn piezoelectric sensor array for gesture input; Journal of Medical and Biomlogical Engineering, Vol. 38, No. 2, pp. 284 295 (2018)

[6] C. Postelnicu, F. Girbacia and D. Talaba: EOGbased visual navigation interface development; $E x$ pert Systems with Applications, Vol. 39, pp. $10857-$ $10866(2012)$

[7] E. Hortal, E. láñez, A. Úbeda, C Perez-Vidal and J. M. Azorín: Combining a brain-machine interface and an electrooculography interface to perform pick and place tasks with a robotic arm; Robotics and Autonomous Systems, Vol. 72, pp. 181-188 (2015)

[8] K. Abe, S. Ohi and M. Ohyama: An eye-gaze input system using information on eye movement history; Universal Access in Human-Computer Interaction, Part II, pp. 721-729 (2007)

[9] D.J. Ward and D.J.C. MacKay: Artificial intelligence: Fast hands-free writing by gaze direction; $\mathrm{Na}$ ture, Vol. 418, No. 6900, p. 838 (2002)

[10] T. E. Hutchinson, K. P. White Jr., W. N. Martin, K. C. Reichert and L. A. Frey: Human-computer interaction using eye-gaze input; IEEE Transactions on Syst. Man. Cybern., Vol. 19, No. 6, pp. 1527-1534 (1989)

[11] H. Sato, K. Abe, S. Ohi and M. Ohyama: A text input system based on information of voluntary blink and eye-gaze using an image analysis; IEEJ Transactions on Electoronics, Information and Systems, Vol. 137, No. 4, pp. 584-594 (2017) (Abstracts in English)

[12] C. M. McCrimmon, J. L. Fu, M. Wang, L. S. Lopes, P. T. Wang, A. Karimi-Bidhendi, C. Y. Liu, P. Heydari, Z. Nenadic and A. H. Do: Performance assessment of a custom, portable, and low-cost braincomputer interface platform; IEEE Trans. Biomed. Eng., Vol. 64, No. 10, pp. 2313-2320 (2017)

[13] K. Hong and M. J. Khan: Hybrid brain-computer interface techniques for improved classification accuracy and increased number of commands: A review; Frontiers in Neurorobotics, Vol. 11, No. 35, pp. 1-27 (2017)
[14] M. Minoda, T. Sugi, Y. Matsuda, S. Goto, T. Yamasaki, A. Oishi and N. Egashira: Development of communication system using eye movement extraction via image processing with blink detection; Proceedings of the 49th ISCIE International Symposium on Stochastic Systems Theory and Its Applications, pp. 21-22 (2017)

[15] E. Watanabe, T. Ozaki and T. Kohama: Analysis of eye movements by interviewer and interviewee in interviews; IEICE Technical Report, H2P2014-43 (2014-9), pp. 1-6 (2014) (Abstracts in English)

[16] G. Bradski and A. Kaehler: Learning OpenCV, O'REILLY, pp. 265-266 (2008)

Takenao SugI

\section{Authors}

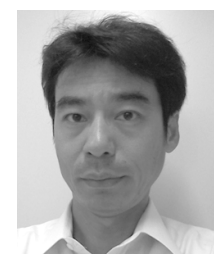

He received his B.E., M.E., and Ph.D. degrees from Saga University in 1992, 1994, and 1999. Since 1995, he has been affiliated with Saga University, where he was a research associate in the Department of Electrical Engineering from 1995 to 2002 , a lecturer from 2002 to 2004 in the Institute of Ocean Energy, Saga University; he has been an associate professor since 2004. His research interests include plant system control and biomedical control. He is a member of IEEE, the Society of Instrument and Control Engineers of Japan, the Japanese Society for Medical and Biological Engineering, Institute of Complex Medical Engineering, the Japanese Society of Sleep Research and the Japanese Society of Clinical Neurophysiology.

Masaya MinodA

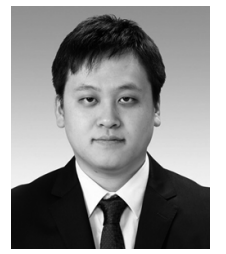

He received his B. E. and M. E. degrees from Saga University in 2016 and 2018. He is currently working at Japan Marine United Cooperation. His research interest is the development of a communication device by image processing.

\section{Yoshitaka Matsuda (Member)}

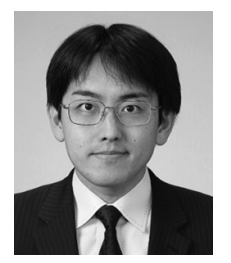

He received his B.E., M.E., and Ph.D. degrees from Kyoto Institute of Technology in 2002, 2004, and 2007, respectively. He worked at Japan Mint from 2007 to 2010 . He is currently an assistant professor at Graduate School of Science and Engineering, Saga University. His research interests include designs of plant mechanical control systems using numerical optimization methods. He is a member of the Institute of Systems, Control and Information Engineers, IEEE, the Society of Instrument and Control Engineers, and the Japan Society of Mechanical Engineers. 


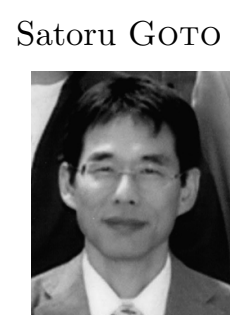

He received his B.E. and M.E. degrees in applied physics and Ph.D. degree from Osaka University in 1988, 1990, and 1995 Since 1990, he has been affiliated with the Faculty of Science and Engineering, Saga University, where he was a research associate in the Department of Electrical Engineering from 1995 to 1996, a lecturer from 1996 to 1998, and an associate professor in the Department of Advanced Systems Control Engineering from 1998 to 2007; he has been a professor since 2007. His research interests include control theory and its applications to real systems. He is a member of the Society of Instrument and Control Engineers of Japan, the Robotics Society of Japan, and the Institute of Systems, Control and Information of Japan, the Japan Society for Precision Engineering, and IEEE.

\section{Naruto EGASHIRA}

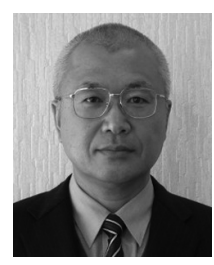

He received his Ph.D. degree from Saga University in 1999. He has been a teacher at Saga Prefectural Taku High School in 1986. Since 1992, he has been affiliated with the Department of Control and Information Systems Engineering, National Institute of Technology, Kurume College, where he was a research associate from 1992 to 1996, a lecturer from 1996 to 2000, an associate professor from 2000 to 2006; he has been a professor from 2006. His research interests include measurement and control using network and mechanical systems control. He is a member of the Society of Instrument and Control Engineers of Japan.

\section{Ayame Oishi}

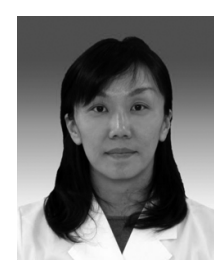

Dr. Oishi has worked at the Department of Neurology and Clinical Neurophysiology, Kyushu University, as a research assistant professor (2009-2015) and a research fellow (2015-present). Based on more than 20 years of herl experience in the fields of clinical neurology and clinical neurophysiology, her main interests are non-invasive assessments by using clinical application of electrophysiological methods in various neurological disorders.

\section{Takao YAmasaki}

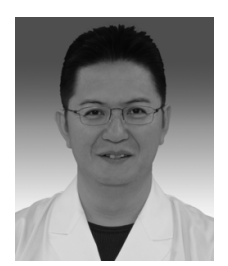

Dr. YAMASAKI is an executive director and a director of Clinical Departments of Minkodo Minohara Hospital. He also worked at the Department of Clinical Neurophysiology, Kyushu University, as an assistant professor (20052007), a research assistant professor (2007-2015) and a research fellow (2015-present). Based on about 20 years of clinical education and experience in the fields of neurology and clinical neurophysiology, his main interest has focused on non-invasive measurements of human brain function, especially higher visual recognition in healthy humans and various neurological disorders, by combining psychophysiological, electrophysiological (EEG, ERPs) and neuroimaging (fMRI, MEG, NIRS) methods. 